\title{
SOME REMARKS ON THE ABSOLUTE MAGNITUDES \\ OF CEPHEIDS
}

\author{
A. OPOLSKI
}

Wroclaw Astronomical Observatory, Wroclaw, Poland

Abstract. This paper contains a rediscussion of the period-luminosity relation of type I-cepheids. Special attention is paid to the radius and its variation.

In my contribution I would like to draw your attention to a parameter which can be used for the classification of the Cepheid variable stars and for more precise determination of the period-luminosity, $\left(P, M_{V}\right)$, relation. Let us start with the formula relating three quantities: $M_{V}, R$ and $F_{V}$

$$
M_{V}=-2.5 \log F_{V}-5 \log R+\text { const. }
$$

We may expect that every $\left(P, M_{V}\right)$ relation should be connected with the corresponding $(P, R)$ and $\left(P, F_{V}\right)$ relations, and that we can try to deduce $M_{V}$ using the radii of Cepheids.

The radii of Cepheids have been calculated many times mostly by means of some modification of Wesselink's method. I have used the following procedure: The observed changes of magnitude in the $V$ and $B$ systems are due to the changes of the fluxes $F_{V}$ and $F_{B}$ and the radius $R$

$$
\begin{aligned}
\Delta V & =-2.5 \Delta \log F_{V}-5 \log \left(1+\frac{\Delta R}{R}\right) \\
\Delta B & =-2.5 \Delta \log F_{B}-5 \log \left(1+\frac{\Delta R}{R}\right) \\
\Delta(B-V) & =2.5 \Delta \log F_{V}-2.5 \Delta \log F_{B} .
\end{aligned}
$$

The radius $R$ and its changes $\Delta R$ are defined by the position of the layer in the atmosphere with a constant value of optical depth. Therefore the last relation is correct when this layer is the same for both optical systems. According to Wesselink's assumption we have for the period of each Cepheid a flux-colour relation: $F_{V}=F_{V}(B-V)$. So for $\Delta(B-V)=0$, also $\Delta \log F_{V}=0$ and consequently $\Delta \log F_{B}=0$. In this case we have

$$
\Delta V=\Delta B=-5 \log \left(1+\frac{\Delta R}{R}\right), \text { for } \Delta(B-V)=0 .
$$

In order to increase the accuracy we have used the relation

$$
\Delta\left(\frac{B+V}{2}\right)=-5 \log \left(1+\frac{\Delta R}{R}\right), \text { for } \Delta(B-V)=0 \text {. }
$$


It seems very convenient to fix one system of the geometric displacements $\Delta R$ obtained from radial velocity integration. Let us measure all $\Delta R$ from $R_{\max }$

$$
\Delta R=R-R_{\max }
$$

Then the expression in formula (6) can be written

$$
5 \log \left(1+\frac{\Delta R}{R_{\max }}\right)=\frac{2.17}{R_{\max }} \Delta R \cdot c,
$$

where the correcting factor $c$ depending on $\Delta R / R_{\max }$ can be calculated with the approximate value of $R_{\max }$ (see Opolski, 1973). Now by introducing the 'rectified displacement' $r$

$$
r=\Delta R \cdot c,
$$

we have the formula

$$
\Delta\left(\frac{B+V}{2}\right)=\Delta m_{R}=-2.17 \frac{\Delta r}{R_{\max }}, \text { for } \Delta(B-V)=0 .
$$

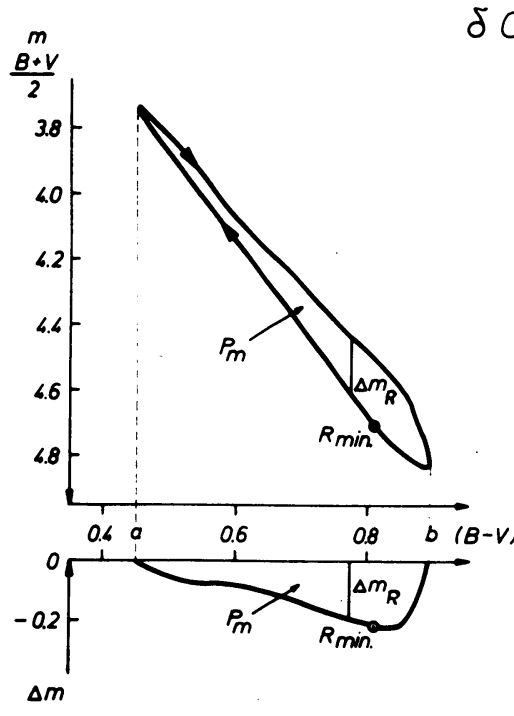

$\delta \mathrm{Cep}$

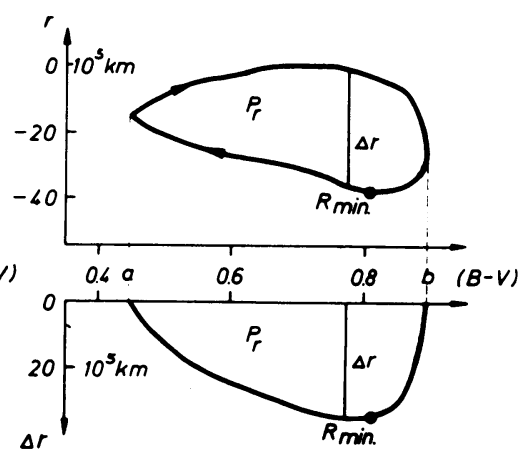

$$
\begin{aligned}
\Delta m_{R} & =-\frac{2.17}{R_{\text {max }}} \Delta r \\
\int_{a}^{b} \Delta m_{R} d(B-v) & =-\frac{2.17}{R_{\text {max. }}} \int_{a}^{b} \Delta r d(B-v) \\
P_{m} & =-\frac{2.17}{R_{\text {max. }}} P_{r}
\end{aligned}
$$

Fig. 1. Photometric relative displacement $\Delta m_{R}$ and geometric displacement $\Delta r$ as functions of $(B-V)$ for $\delta$ Cep. 
This relation can be represented graphically (see Figure 1). Let us draw a loop described by a star on the diagram with the axes $(B+V) / 2$ and $(B-V)$. The width of the loop measured perpendicular to the $(B-V)$ axis is determined by the points with equal $(B-V)$, so for them $\Delta(B-V)=0$ and $\Delta((B+V) / 2)$, denoted by $\Delta m_{R}$, is just the value occurring in the formula (9). This value represents the photometric relative displacement resulting from the geometric one $\Delta R / R_{\max }$ and expressed on the magnitude scale.

Similarly, we can draw a loop on the $r$ vs. $(B-V)$ plane and determine the difference $\Delta r$ for the same value of $(B-V)$. So we obtain the value of $R_{\max }$ from the formula (9)

$$
R_{\max }=-2.17 \frac{\Delta r}{\Delta m_{R}}
$$

This calculation can be repeated for every value of $(B-V)$ or, using the whole areas of the loops, $P_{m}$ and $P_{r}$ :

$$
\begin{aligned}
& P_{m}=\int_{a}^{b} \Delta m_{R} \mathrm{~d}(B-V), \quad P_{r}=\int_{a}^{b} \Delta r \mathrm{~d}(B-V) \\
& R_{\max }=-2.17 \frac{P_{r}}{P_{m}} .
\end{aligned}
$$

In order to make the photometric relative displacement $\Delta m_{R}$ more easy to observe and to compare with other stars, we plot the values $\Delta m_{R}$ in the lower part of the diagram as a function of $(B-V)$. Similarly the corresponding values of $\Delta r$ are to be seen below the loop $P_{r}$. Both curves should have the same shape and differ only in the scales of $\Delta m_{R}$ and $\Delta_{r}$.

The values of maximum radius can also be calculated by the numerical method presented already by the author (Opolski, 1973). In this method, the general relation between flux and colour introduced by Wesselink is specified in the form:

$$
-2.5 \Delta \log F_{V}=k \Delta(B-V) \text {, }
$$

where $k$ is a constant. The correctness of this assumption can be proved for the majority of Cepheids (see Figure 2). But some of them, e.g. SV Vul, do not follow this relation. As a consequence of equation (13), we have

and

$$
\begin{aligned}
& -2.5 \Delta \log F_{B}=(k+1) \Delta(B-V), \\
& -2.5 \Delta\left(\frac{\log F_{V}+\log F_{B}}{2}\right)=(k+0.5) \Delta(B-V)
\end{aligned}
$$

$$
\Delta \log F_{V}=\frac{k}{k+1} \Delta \log F_{B}
$$

Therefore in our computations we have used the formula

$$
\Delta\left(\frac{B+V}{2}\right)=(k+0.5) \Delta(B-V)-2.17 \frac{\Delta r}{R_{\max }},
$$




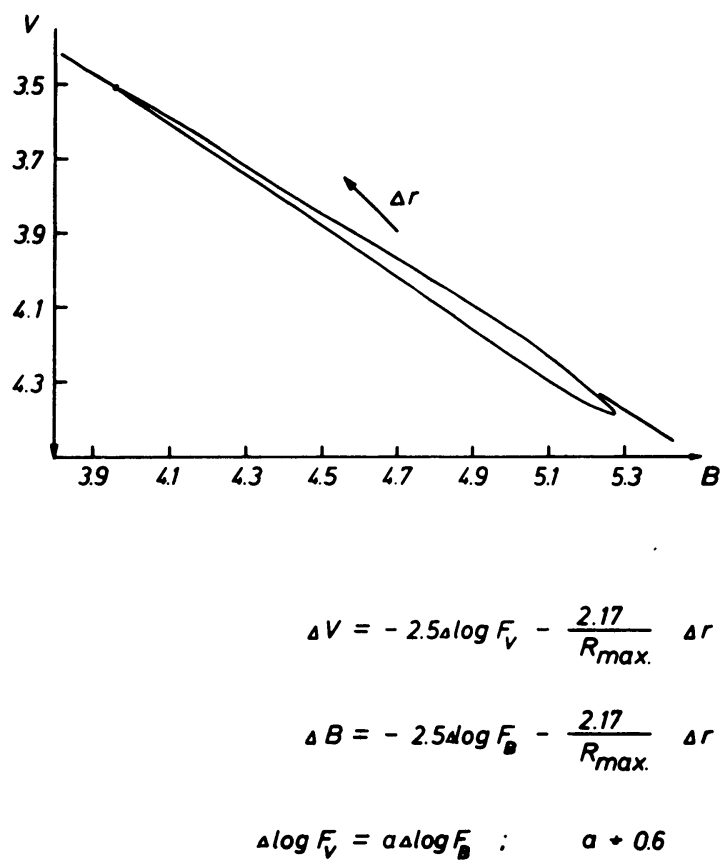

Fig. 2. The $V$ vs. $B$ diagram for $\delta$ Cep shows the linear relation: $\Delta \log F_{V}=a \Delta \log F_{B}$. The loop is caused by the contribution of the displacement $\Delta r$.

where the differences between the individual values and their means have been introduced.

For each star a set of 20 equations for phases $0.00,0.05, \ldots, 0.95$ has been solved and the two unknown quantities $k$ and $R_{\max }$ determined. Then the phases of $\Delta r$ were shifted and the set of equations solved again. This procedure was repeated until the best accuracy was obtained. 18 stars with reliable radial velocities and photometry have been treated in this way. All numerical data are listed in Table I with the following notation: $\log R_{\max }$ is the logarithm of $R_{\max }$ expressed in $\mathrm{km} ; \log A(R)$ the logarithm of the amplitude of the geometric displacement $\Delta R$ in $\mathrm{km} ; A(R) / R_{\max }$ the geometric relative displacement; $\overline{\Delta m_{R}}$ the mean photometric relative displacement

$$
\overline{\Delta m_{R}}=\frac{P_{m}}{A(B-V)}=\frac{P_{m}}{b-a}
$$

and $A(B-V)$ the amplitude of the $(B-V)$ variation. 
TABLE I

Radii and relative displacements of Cepheids

\begin{tabular}{|c|c|c|c|c|c|c|}
\hline * & $\log P$ & $\log R_{\max }$ & $\log A(R)$ & $\frac{A(R)}{R_{\max }}$ & $\overline{\Delta m_{R}}$ & $A(B-V)$ \\
\hline \multicolumn{7}{|c|}{ Group 0} \\
\hline RT Aur & 0.571 & 7.935 & 6.316 & 0.024 & $0 m 038$ & $0^{m} 41$ \\
\hline T Vul & 0.647 & 7.824 & 6.439 & 0.041 & 0.085 & 0.33 \\
\hline FF Aql & 0.650 & 7.858 & 6.167 & 0.020 & 0.042 & 0.16 \\
\hline S Cep & 0.730 & 7.696 & 6.548 & 0.071 & 0.093 & 0.45 \\
\hline \multicolumn{7}{|c|}{ Group I } \\
\hline U Aql & 0.847 & 7.497 & 6.681 & 0.153 & 0.173 & 0.36 \\
\hline$\eta$ Aql & 0.856 & 7.678 & 6.666 & 0.097 & 0.167 & 0.43 \\
\hline W Sgr & 0.881 & 7.659 & 6.690 & 0.107 & 0.160 & 0.43 \\
\hline W Gem & 0.898 & 7.588 & 6.692 & 0.128 & 0.197 & 0.37 \\
\hline S Sge & 0.923 & 7.728 & 6.714 & 0.097 & 0.138 & 0.42 \\
\hline$\beta$ Dor & 0.993 & 7.785 & 6.763 & 0.095 & 0.142 & 0.37 \\
\hline$\zeta$ Gem & 1.007 & 7.733 & 6.684 & 0.089 & 0.094 & 0.29 \\
\hline X Cyg & 1.214 & 7.968 & 7.240 & 0.187 & 0.306 & 0.58 \\
\hline T Mon & 1.432 & 8.124 & 7.425 & 0.200 & 0.268 & 0.60 \\
\hline l Car & 1.551 & 8.164 & 7.387 & 0.167 & 0.294 & 0.48 \\
\hline SV Vul & 1.654 & 8.185 & 7.629 & 0.278 & 0.264 & 0.69 \\
\hline \multicolumn{7}{|c|}{ Group II } \\
\hline$\kappa \mathrm{Pav}$ & 0.958 & 7.371 & 6.681 & 0.204 & 0.326 & 0.50 \\
\hline Y Oph & 1.233 & 7.530 & 6.709 & 0.151 & 0.115 & 0.29 \\
\hline W Vir & 1.237 & 7.597 & 7.137 & 0.346 & 0.633 & 0.53 \\
\hline
\end{tabular}

The $\left(P, R_{\max }\right)$ relations represented in Figure 3 show a complicated structure. One can distinguish three regions: The $\left(P, R_{\max }\right)$ relation for classical Cepheids, marked as I, is defined by the stars near $\log R_{\max }=7.7$ and $\log P=0.9$ and reaches to $\log R_{\max }=$ $=8.2$ for $\log P=1.6$. Below are situated two typical stars of population II, $\mathrm{W}$ Vir and $\kappa$ Pav, and unexpectedly also Y Oph. They have radii smaller by $\Delta \log R_{\max }=0.4$ than the Cepheids in region I. But above the typical Cepheids I there are 3 stars with radii greater by about $\Delta \log R_{\max }=0.3$. This group has been denoted as type 0 . For these stars the radii seem to diminish as the periods increase. The star $\delta$ Cep falls near this group.

All the stars in Figure 3 are labelled with the corresponding values of $A(R) / R_{\max }$. Taking these numbers into consideration we can say that, for a given period, the greater $R_{\max }$ the smaller $A(R) / R_{\max }$. So we can easily distinguish the three $\left(P, R_{\max }\right)$ relations from each other by means of the values $A(R) / R_{\max }$ which change in a characteristic manner.

The geometric relative displacements $A(R) / R_{\max }$ can be obtained only for stars with known radial velocities. The number of these stars is very limited and there is no hope 
that the situation in this respect will soon improve. On the other hand, the number of accurate photometric measurements is increasing continuously and it would be very advantageous to get more information from these data. Therefore, we tried to replace the geometric relative displacement, $A(R) / R_{\max }$, by the mean photometric one, $\overline{\Delta m_{R}}$.

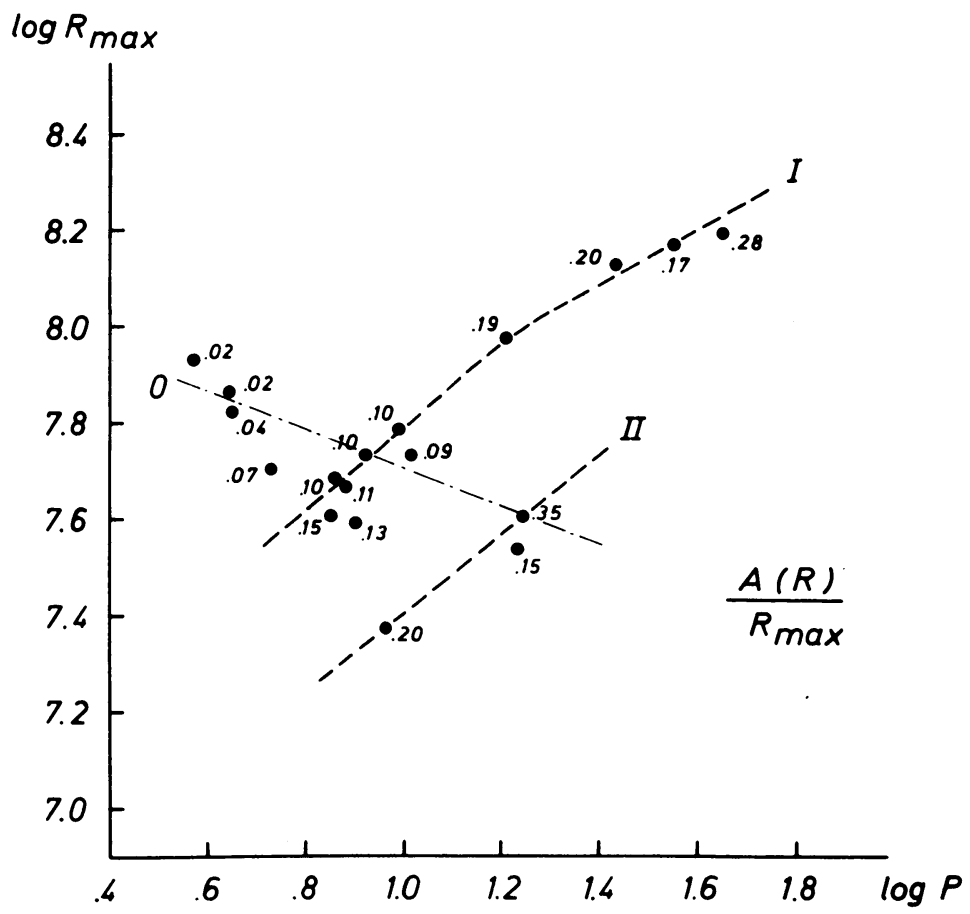

Fig. 3. Period-radius relations for Cepheids with the separation of the 0 , I and II groups. The stars are labelled with the amplitude of their relative geometric displacement $A(R) / R_{\max }$.

From the comparison of both values, it is possible to establish a relation

$$
\overline{\Delta m_{R}}=1.5 \frac{A(R)}{R_{\max }} .
$$

Figure 4 shows photometric relative displacements for three stars belonging to groups $0, I$ and II. It can be seen that these quantities and their mean values follow the changes of $A(R) / R_{\max }$

\begin{tabular}{|c|c|c|c|}
\hline Group & 0 & I & II \\
\hline Star & T Vul & S Sge & W Vir \\
\hline$\frac{A(R)}{R_{\max }}$ & 0.04 & 0.11 & 0.35 \\
\hline$\overline{\Delta m_{R}}$ & 0.08 & 0.14 & 0.63 \\
\hline
\end{tabular}


T Vul
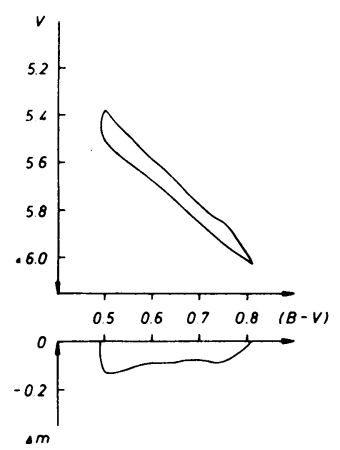

$\begin{array}{cc}\log P & 0.667 \\ \log R_{\text {max }} & 7.826 \\ \frac{A(R)}{R_{\text {max }}} & 0.061\end{array}$

\section{S Sge}
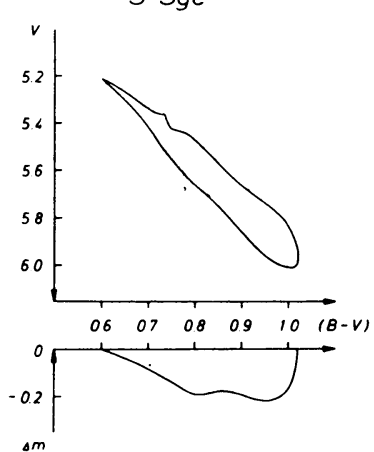

0.881

7.659

0.107
W Vir
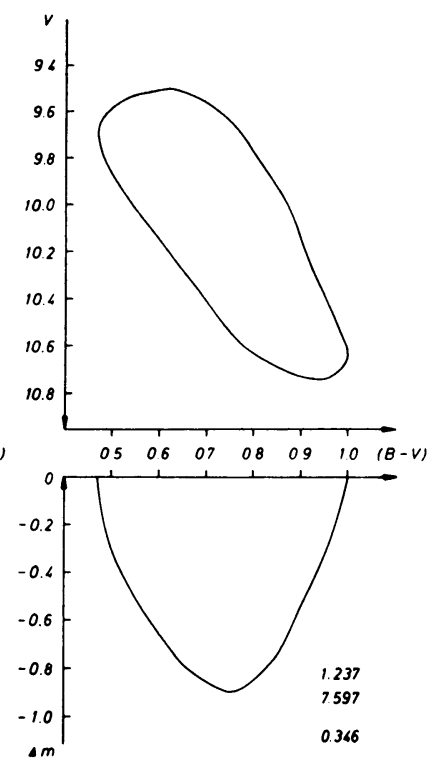

Fig. 4. The photometric relative displacements for Cepheids of 0 , I and II group. The stars are situated on the line running across the $\left(P, R_{\max }\right)$ relations in Figure 3.

So it seems to be possible to use the value of $\overline{\Delta m_{R}}$ which can be obtained on the basis of the photometry alone as an additional parameter in order to specify better the properties of individual Cepheids.

As an application of the parameter $\overline{\Delta m_{R}}$, we can test that the Cepheids belonging to open clusters and used for absolute magnitude determinations have values of $\overline{\Delta m_{R}}$ typical for group I (Table II). We can combine the values of radii and absolute magnitudes according to Sandage and Tammann (1968) and Kukarkin (1970) to derive a

TABLE II

Absolute magnitudes and mean photometric relative displacements of Cepheids in open clusters

\begin{tabular}{lccc}
\hline$*$ & $\log P$ & $\langle M\rangle_{V}$ & $\overline{\Delta m_{R}}$ \\
\hline EV Sct & 0.490 & -2.6 & 0.051 \\
SZ Tau & 0.498 & -2.9 & 0.082 \\
CF Cas & 0.688 & -3.1 & 0.110 \\
VY Per & 0.730 & -3.5 & 0.056 \\
U Sgr & 0.829 & -3.9 & 0.145 \\
DL Cas & 0.903 & -3.8 & 0.131 \\
S Nor & 0.989 & -4.0 & 0.133 \\
VX Per & 1.037 & -4.3 & 0.154 \\
SZ Cas & 1.134 & -4.7 & 0.096 \\
$l$ Car & 1.551 & -5.9 & 0.294 \\
RS Pup & 1.617 & -6.1 & 0.270
\end{tabular}


period-radius-luminosity relation with the parameter $\overline{\Delta m_{R}}$ which can help in assigning the individual stars to different groups. But with known $R_{\max }$ and $M_{V}$ it is possible to get the fluxes $F_{V}$ using the formula given by Allen (1963),

$$
\log F_{V}=-0.4 M_{V}+8.87-2 \log R / R_{\odot},
$$

where $F_{V}$ is expressed in $\mathrm{erg} \mathrm{cm}^{-2} \mathrm{~s}^{-1} \AA^{-1}$.

The complete period-radius-luminosity-flux relation is represented in the Table III, where $R_{\text {max }}, M_{V}$ and $F_{V}$ can be regarded as functions of $P$ and $\overline{\Delta m_{R}}$.

\section{TABLE III}

Period-luminosity-radius-flux relations for Cepheids

\begin{tabular}{|c|c|c|c|c|}
\hline $\log P$ & $\Delta m_{R}$ & $\langle\boldsymbol{M}\rangle_{\boldsymbol{V}}$ & $\log R_{\max }$ & $\log F_{V}$ \\
\hline & \multicolumn{4}{|c|}{ Group 0} \\
\hline 0.6 & 0.04 & -4.5 & 7.90 & 6.56 \\
\hline \multirow[t]{2}{*}{0.7} & 0.04 & -4.0 & 7.75 & 6.66 \\
\hline & \multicolumn{4}{|c|}{ Group I } \\
\hline 0.4 & 0.06 & -2.6 & 7.15 & 7.30 \\
\hline 0.6 & 0.09 & -3.1 & 7.44 & 6.93 \\
\hline 0.8 & 0.13 & -3.6 & 7.60 & 6.82 \\
\hline 1.0 & 0.16 & -4.2 & 7.80 & 6.64 \\
\hline 1.2 & 0.20 & -4.8 & 7.98 & 6.51 \\
\hline 1.4 & 0.25 & -5.4 & 8.08 & 6.54 \\
\hline \multirow[t]{2}{*}{1.6} & 0.29 & -5.9 & 8.20 & 6.53 \\
\hline & \multicolumn{4}{|c|}{ Group II } \\
\hline 0.9 & 0.30 & -1.8 & 7.30 & 6.68 \\
\hline 1.1 & 0.45 & -2.1 & 7.48 & 6.44 \\
\hline 1.3 & 0.60 & -2.5 & 6.64 & 6.28 \\
\hline
\end{tabular}

\section{References}

Allen, C. W.: 1963, Astrophysical Quantities, 2nd ed., London.

Kukarkin, B. W.: 1970, Pulsating Stars, Moscow, p. 126.

Opolski, A.: 1973, in B. Hauck and B. E. Westerland (eds.), 'Problems of Calibration of Absolute Magnitudes, and Temperature of Stars', IAU Symp. 54, 165.

Sandage, A. R. and Tammann, G. A.: 1968, Astrophys. J. 157, 683. 